\title{
Design of Linear Array for Shaped Beams using Enhanced Flower Pollination Optimization Algorithm for Marine Radar Applications
}

Nagavalli Vegesna

Annamalai University

Yamuna G

Annamalai University

Sudheer Kumar Terlapu ( $\nabla$ profsudheer@ieee.org )

Shri Vishnu Engineering College For Women https://orcid.org/0000-0003-4535-6599

\section{Research Article}

Keywords: Enhanced Flower Pollination Algorithm, Linear array, Sidelobe level

Posted Date: August 26th, 2021

DOI: https://doi.org/10.21203/rs.3.rs-807366/v1

License: (c) (i) This work is licensed under a Creative Commons Attribution 4.0 International License.

Read Full License 


\title{
Design of Linear Array for Shaped Beams using Enhanced Flower Pollination Optimization Algorithm for Marine Radar Applications
}

\author{
Nagavalli Vegesna ${ }^{1}$,G.Yamuna ${ }^{2}$, T.Sudheer Kumar $^{3}$ \\ ${ }^{1,2}$ Annamalai University, Chidambaram,Tamilnadu, INDIA \\ ${ }^{3}$ Shri Vishnu Engineering College for Women (Autonomous), Bhimavaram, AP, INDIA \\ \{Mail id: nagavalli.v@ srkrec.ac.in*1, yamuna.sky@gmail.com2, profsudheer@ieee.org3\}
}




\title{
Design of Linear Array for Shaped Beams using Enhanced Flower Pollination Optimization Algorithm for Marine Radar Applications
}

\author{
Nagavalli Vegesna ${ }^{1}$,G.Yamuna ${ }^{2}$, T.Sudheer Kumar ${ }^{3}$ \\ ${ }^{1,2}$ Annamalai University, Chidambaram,Tamilnadu, INDIA \\ ${ }^{3}$ Shri Vishnu Engineering College for Women (Autonomous), Bhimavaram, AP, INDIA \\ \{Mail id: nagavalli.v@ srkrec.ac.in*1, yamuna.sky@gmail.com, profsudheer@ieee.org3\}
}

\begin{abstract}
In order to generate narrow beams with individual heights of sidelobes, we perform a synthesis of sum patterns. The side lobes can be adjusted to any arbitrary specification and flat top far field radiation patterns using Enhanced Flower Pollination Algorithm (EFPA) is presented in this paper. The problem of designing an array can be expressed as an optimization problem that aims to reduce the first two sidelobes to $-50 \mathrm{~dB}$ and by reducing the remaining sidelobes to $-40 \mathrm{~dB}$. The EFPA is applied to determine the excitation coefficients of their amplitudes so as to obtain the required pattern. The objective for case 2 is to obtain the flat top sector beam for specified angular regions. Complex excitation coefficients are determined using EFPA for various beam widths. The radiation patterns are generated numerically using the obtained excitation coefficients. The results reveal that not only the ripples are controlled in the trade-in region, but also maintained sidelobe levels in the acceptable limits. The results presented are very much useful for marine radar applications.
\end{abstract}

Index Terms —Enhanced Flower Pollination Algorithm, Linear array, Sidelobe level.

\section{Introduction:}

There has been a lot of technological development in the communication era compared to a generation of $2 \mathrm{G}$ to $5 \mathrm{G}$ which includes speed, range, etc. To increase the range of communication, antennas have played a prominent role. For long-distance communication, Array Antennas are used to increase the gain. Due to a lot of development in communication, there is an increase in traffic and an increase in the interference of the signals. To avoid this there should be a reduction in the levels of the side lobes and the reduction of nulls in a preferred way. The key feature of modern-day communication lies in designing an accurate model and synthesis of antenna arrays. The antenna's radiation pattern relies on various array parameters of antennas, like the geometrical arrangement of the array, the placement gaps between elements, and their respective amplitude weights. Due to the hiking need of lossless and fast communication, there is a need to develop a much effective array pattern of antennas that has a relatively minimized portion in the levels of side lobes and smaller first null beamwidth. From the past two decades, there are a lot of developments in the naturally Inspired Algorithms to obtain nulls in the preferred direction, optimization of side lobe levels along with first null beam width and beam shaping.

Cat swarm optimization (CSO) which aims in the reduction of the levels of sidelobes and Directivity improvement. The results of the design thus mentioned has a greater reduction in sidelobe level compared to the uniformly excited array pattern. Also, we can observe the increase in sideband frequency, decrease in the radiated power from both the harmonic frequencies and sideband levels (SBL) (Ram et al. 2015). 
To minimize side lobe levels by maintaining beam width, the values of weights are optimized for each antenna element using a Genetic Algorithm(GA). The results obtained with GA when compared with conventional methods, GA performs better by getting optimal results (Zhang et al. 2014) .

Ant Lion Optimization(ALO) algorithm is implemented based on the behavior of an ant lion insect on how it hunts for prey. This process modeled mathematically. ALO optimizes the nonlinear values of amplitude excitation for concentric CAA to obtain lower side lobe level with narrow FNBW excluding the effect of mutual coupling. This, in turn, reduces the effect of interference due to SLL and a genetic approach that is applicable to different types of antenna elements with the help of a pattern multiplication technique (Das et al.2019).

To increase the reliability of a communication system, one factor that is to be mainly considered is the suppression of the levels attained by the side lobes. The amalgamation of the Antlion Optimization algorithm and Grasshopper Optimization Algorithm gives a novel hybrid algorithm which is the objective of this study. The main goal of the algorithm presented in the paper is to rectify the drawbacks of ALO and GOA. The results display the improvement of a circular antenna array in optimizing its radiation pattern along with a fast convergence rate(Amairehet al. 2019).

Cuckoo search (CS) algorithm is used for implementing N-dimensional optimization issues. It works with very few parameters and is easy to understand compared to other nature-inspired algorithms. Visibly better performance of the CS can be observed when compared to the standard evolutionary algorithms. A part of the worst solutions is ignored and update with the latest solution. It is a powerful optimization technique due to its simplicity and robustness. Also for functions like Griewank, Rastigrin, and Rosenbrock, CS Algorithmis better as it has a high rate of convergence rate and fewer chances of local minima (Khodieret al. 2013).

The study presents a cuckoo search-based chicken swarm optimization (CSCSO) which is designed to optimize the linear and circular antenna arrays in terms of their amplitude. It is an amalgamation of the efficient overall searchability of CS and the advantage of the hierarchical performance of the CSO.CSCSO has the capability to generate low levels of side lobes provided a fixed main lobe width. The simulation results also show a considerate increase in accuracy and convergence rate for CSCSO compared to the standard CSO, CS, and PSO algorithms (Liang et al. 2017).

An evolutionary algorithm which is used to solve the problems of multi-objective optimizations. Flower pollination algorithm (FPA) is implemented to a linear array to result in a side lobe level minimization and deep nulls detection. The results state that FPA out performs the algorithms in comparison such as particle swarm optimizationtechnique, ant colony optimization technique and cat swarm optimization or sometimes yields a similar performance. The comparative results of the standard arrays and the modified arrays that are optimized using these algorithms showcase that the FPA performs well in achieving a peak suppression of the side lobe level by parallel placement of strong nulls in the direction of interest.(Saxena et al. 2016).

Reduced SLL, HPBW, directivity and desired null placement by optimizing excitation of amplitude and spacing between elements can be obtained using Particle Swarm Optimization Algorithm. The results when compared with other optimization algorithms like Real Code Genetic Algorithm and Biogeograpic Based Optimization, the PSO has a better reduction in SLL, HPBW and null placement.(Rahman et al. 2017).

For optimization the algorithms TA,GA and PSO are used for many engineering applications. When these algorithms are combined to make hybrid model TAGAPSO has best results of optimization 
than individual algorithms. TAGAPSO gives best results of suppressed SLL and HPBW of the linear antenna array.(Yigit et al. 2018).

To overcome challenges in upcoming $5 \mathrm{G}$ communication for high gain and to achieve higher capacity, large scale antennas required. At the same time complexity of hardware, power, cost should be reduced. To achieve this Atom search optimization is best solution with minimized side lobe levels, desired beam forming, null depth and good convergence (Almagboul et al. 2019).

Beam pattern synthesizing problem of circular and linear antenna array can be formulated by Invasive Weed Optimization by reducing side lobe levels of beam patterns. It has best results for different antenna array elements like 8,16 and 32 (Sun and Liu 2018).

The problem of side lobe power level increase due to element failure of antenna array can be addressed using Firefly Algorithm(FA). The error between failed and prefailed side lobe pattern can be reduced using FA(Grewal et al. 2012). The improved version of FA is Enhanced Firefly Algorithm(EFA). It has high convergence rate and provides minimized side lobe levels than FA(Yoshimoto et al. 2019).

Spider monkey optimization(SMO) is best suited for optimization problemsin engineering. In this binary spider monkey optimization(binSMO), the place of every spider monkey has logic values of ' 0 ' and '1' which are applied for CCAA thinning. The result of the algorithm performed well for different rings of concentric circular antenna array technique which is stated as an comparison with DEGL,BBO etc. The reduction in SLL has been upto $27 \%$ when compared to DEGL which can be stated great improvement acquired by using binSMO. This method is an effective binary optimization technique due to fast convergence(Singh et al. 2016).

In elliptical and concentric elliptical antenna array to reduce side lobe levels, a novel optimization technique called Gray Wolf Optimization(GWO) is used. It depends on hunting process of gray wolf. Including element excitation another parameters is also considered that is eccentricity for performance of array to optimize SLL (Recioui et al. 2019).To impose nulls in desired direction to avoid interference in uniformly spaced half wave dipole linear antenna, a optimization algorithm called Bat Algorithm(BA) is discussed (Van Luyen et al. 2017).

Whale Optimization Algorithm is first stated in 2016 by Mirjalili and Lewis (Mirjali et al. 2016). It is completely depend on humpback whale hunting. WOA has better null steering and reduced side lobe levels when applied to aperiodic linear array. When compared with PSO, CLPSO, IWO/WDO and differential evolution, WOA has best radiation pattern results(Zhang et al. 2018).

With the inspiration of clonal selection theory of human, a algorithm developed is Clonal Selection Algorithm(CLONALG) and does not have complex mathematical equations. Position of a linear array can be controlled to achieve steering of nulls in the preferred way. Side lobe levels, level of null depth can be controlled and Multiple nulls can be imposed to avoid interference using CLONALG (Guney et al.2007) . It is also used for reconfigurable dual beam linear antenna array (Akdagli et al. 2007).

Tabu Search Algorithm(TSA) best suited for linear antenna array which is uniformly distributed to reduce side lobe levels with fixed beam width. TSA is proposed by Glover in 1986. Tabu search establish an optimum set of weights to reduce side lobe levels with fixed beam width. It finds better nearest optimal solution for best optimization (Merad et al. 2008). 


\section{DESIGN FORMULATION:}

\section{A. Array factor}

In a linear array geometry, all the elements are placed along a straight line. An array factor is expressed as the combined product of the elemental and spatial factors. The linear array elements are isotropic whose azimuthal and elevation angles have uniform radiation pattern. Despite of their uniform radiation patterns, they behave in different patterns when they are placed as an array.

The excitation of these elements is concentrated along the center of the array. If we consider an even numbered linear array, its array factor can be formulated as,

$$
\begin{aligned}
& E(\theta)=2 \sum_{i=1}^{M} A_{i} \operatorname{Cos}[\pi(i-0.5) v] \\
& \mathrm{v}=\sin \theta
\end{aligned}
$$

Where,

The broadside and line of observer between angle is $\theta$

$i^{\text {th }}$ element current excitation is $A_{i}$

Number of Elements are M

The radiation pattern of the linear array is majorly dependent on parameters like excitation amplitude, the elemental spacing and their phases. To obtain the desired radiation pattern we must maintain proper amplitude distribution.

\section{B. Fitness Function}

Case 1:

The Fitness function for observing varying SLL with respect to first two SLLs immediate to main beam called as close in SLLs and SLLs aw ay from the main beam is given as

$$
\begin{aligned}
& f f 1_{\max }=\max \left(S L L_{1}, S L L_{2}\right) \\
& \text { and } \\
& f f 2_{\text {max }}=\max \left(A F\left(-\theta\left(S L L_{2}\right)>A F(\theta)>A F\left(\theta\left(S L L_{2}\right)\right)\right]\right. \\
& \text { where }-90<\theta<90 \\
& f 1=f f 1_{\text {max }}+S L L_{c} \quad \text { if } \quad f f 1_{\text {max }}>-S L L_{c} \\
& =0 \quad \text { otherwise } \\
& f 2=f f 2_{\text {max }}+S L L_{f} \quad \text { if } \quad f f 2_{\text {max }}>-S L L_{f} \\
& =0 \quad \text { otherwise }
\end{aligned}
$$

$\mathrm{SLL}_{1}, \mathrm{SLL}_{2}$ are side lobe levels of 1st and 2nd side lobes immediate to main lobe.

$-\mathrm{SLL}_{\mathrm{c}}$ refers to desired close in SLL which is $-50 \mathrm{~dB}$ in this case 
$-\mathrm{SLL}_{\mathrm{f}}$ refers to desired other side lobes level which is $-40 \mathrm{~dB}$ in this case Equation (3) is the reason for minimizing close in side lobe levels and Equation (4) takes the role of maintaining other SLLs at -40dB. A cost enhancement factor which is simply a numerical value can be added to ff 1 to compete with the ff 2 . This is the obvious case of increasing the cost of close in side lobe levels anticipating fast convergence to the desired level. The envelope of the desired pattern is given in the Fig. 1 and Fig.2.

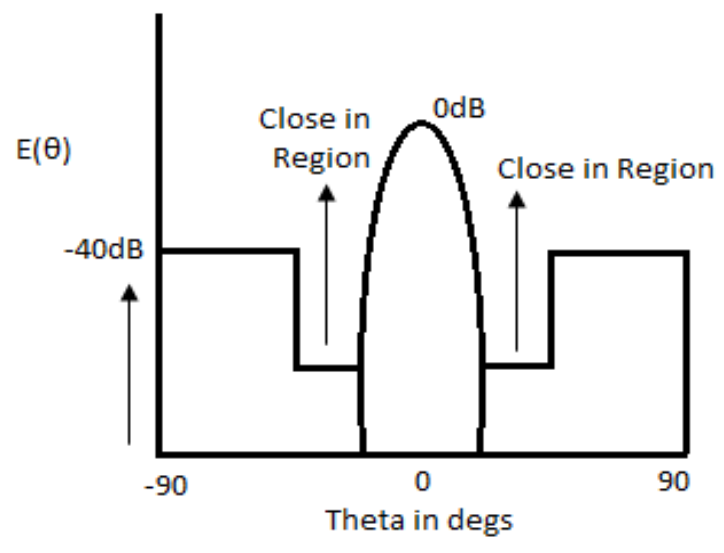

Fig. 1: Envelope of the desired pattern

\section{Case 2:}

With the requirement to achieve a main beam with flat top and minimized side lobe level, a suitable fitness function is developed as below.

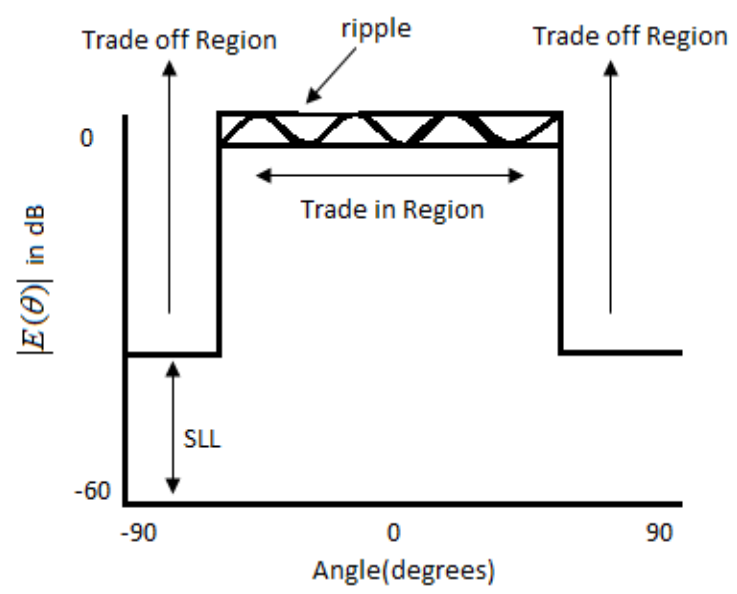

Fig. 2: Desired Radiation Pattern

Keeping in knowledge of the above discussion, a generalized fitness function is generated as,

For $\theta_{l} \leq \theta \leq \theta_{h}$

$f_{1}=\sum_{\theta=\theta_{1}}^{\theta_{\mathrm{h}}}|E(\theta)| \quad$ if $E(\theta) \geq E_{\mathrm{sec}}$

For $\theta \leq \theta_{l}$ and $\theta \geq \theta_{h}$ 


$$
\begin{aligned}
& f_{2}=\sum_{\theta=-\frac{\pi}{2}}^{\theta_{1}}\left(E(\theta)+S L L_{\text {opt }}\right)+\sum_{\theta=\theta_{h}}^{\frac{\pi}{2}}\left(E(\theta)+S L L_{\text {opt }}\right) \\
& \operatorname{ifE}(\theta) \geq-S L L_{\text {opt }}
\end{aligned}
$$

Finally, cost function is now written as

$$
f_{\mathrm{sec}}=f_{1}+f_{2}
$$

Where $\mathrm{SLL}_{\mathrm{opt}}$ and $\mathrm{E}_{\mathrm{sec}}$ are the maximum sidelobe level we aim for and the main beam ripple obtained respectively. $\theta_{\mathrm{l}}$ to $\theta_{\mathrm{h}}$ is the angular range of the total sector pattern. We used $\mathrm{SLL}_{\mathrm{opt}}=25 \mathrm{~dB}$ and $\mathrm{E}_{\mathrm{sec}}=1.0$ $\mathrm{dB}$. The vector $\mathrm{c}$ is a combination of amplitude along with the phase variables. $\mathrm{E}_{\mathrm{sec}}$ is the control parameter which is used to obtain flat beam. Whereas, to establish control over the bandwidth SLL $_{\text {opt }}$ plays an important role. The values of $\mathrm{SLL}_{\mathrm{opt}}$ and $\mathrm{E}_{\mathrm{sec}}$ have to be selected so as to match the trade-off between the beamwidth, ripple magnitude and the side lobe level in the trade in regions.

\section{ENHANCED FLOWER POLLINATION ALGORITHM}

To avoid slow and premature convergence of Flower Pollination Algorithm(FPA) which leads to global optimum can be overcome by EFPA. EFPA aims for modifications to FPA which include Cauchy based global pollination, Enhanced local pollination and Dynamic switching probability.

a)Cauchy Based Global Pollination:

Cauchy based operator $(\delta)$ is used in this phase. Cauchy random variable operator with distribution is given by

$$
\delta=(1 / 2)+((1 / \pi) \arctan (x / h))
$$

The density function of Cauchy ,

$$
f_{\text {cauchy }(0, g)}(x)=(1 / \pi) \arctan \left(h /\left(h^{2}+x^{2}\right)\right)
$$

Global pollination general equation,

$$
x_{i}^{t+1}=x_{i}^{t}+c(\delta)\left(R_{*}-x_{i}^{t}\right)
$$

where $\mathrm{h}$, is a scale parameter with value 1 . By using Cauchy operator, premature convergence can be avoided.

b)Enhanced Local Pollination: given by

New pollinator is updated based on local experience and current best pollinators. The equation is

$$
x_{i}^{t+1}=x_{i}^{t}+c\left(R_{*}-x_{i}^{t}\right)+d\left(x_{j}^{t}-x_{k}^{t}\right)
$$

where $\mathrm{c}$ and $\mathrm{d}$ are distributed uniformly random numbers in the range of $\mathrm{j}, \mathrm{k}[0,1]$. jth flower pollinator is different from kth flower pollinator to enhance the local search capabilities.

c)Dynamic Switching Probability:

For more intensive local and global search, dynamic switching probability is used. General formula for switch probability.

$$
p=p-(N-s / N) *(0.1)
$$

where $\mathrm{N}$ is the maximum iterations and $\mathrm{s}$ is the current iteration. 


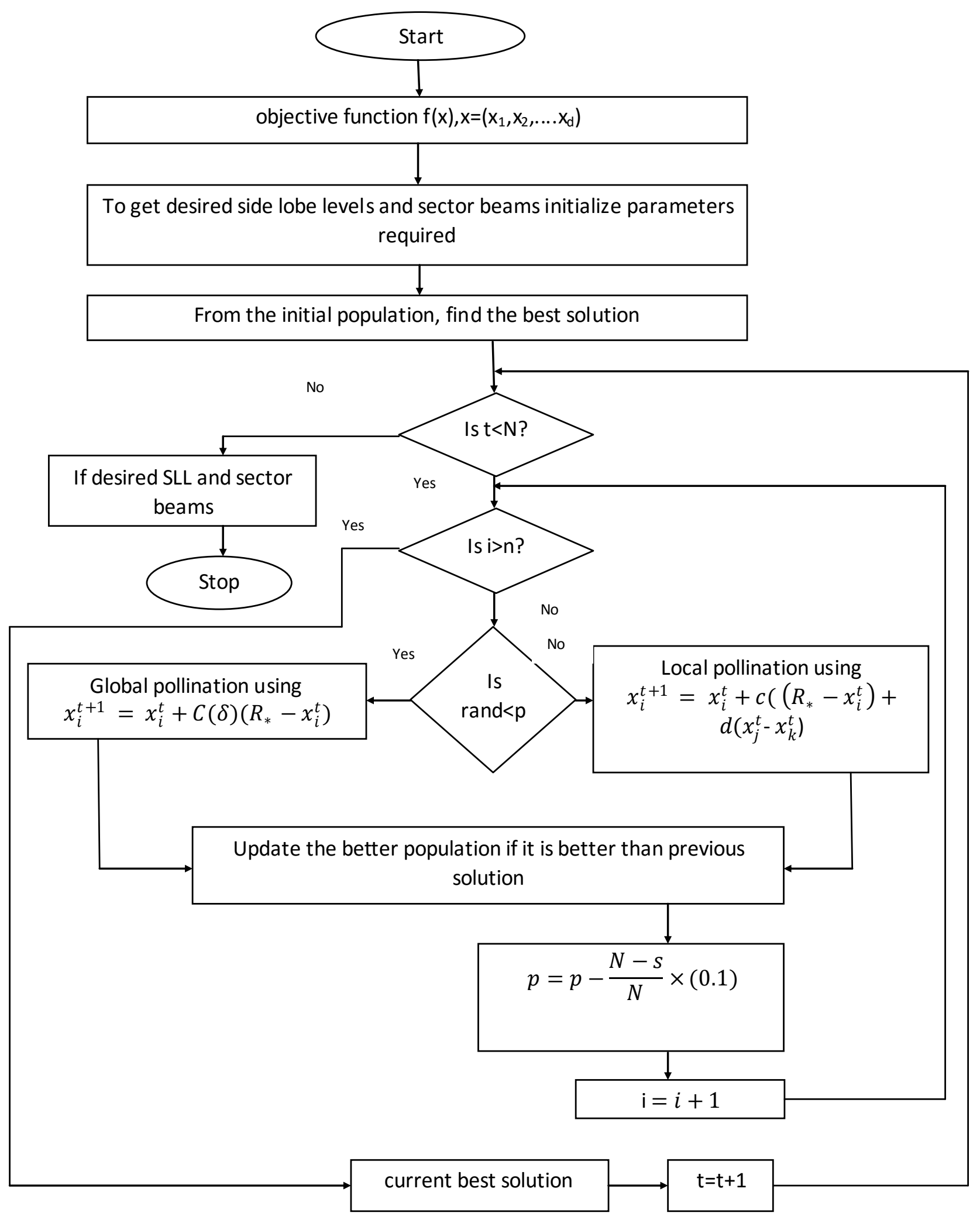

Fig 3: Flow Chart for EPFA. 


\section{RESULTS}

In case 1, Amplitude excitations obtained using EFPA algorithm, yields the optimized radiation patterns with a reduced close-in side lobes to $-50 \mathrm{~dB}$ and the remaining side lobes to $-40 \mathrm{~dB}$. Computations are repeated for number of elements equal to 10 to 50 in steps of 20 and are presented in figures [4-9]

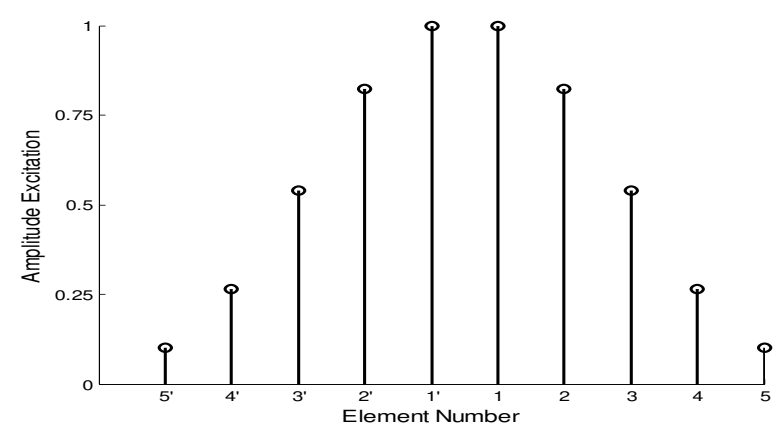

Fig 4: 10 elements Amplitude Distribution.

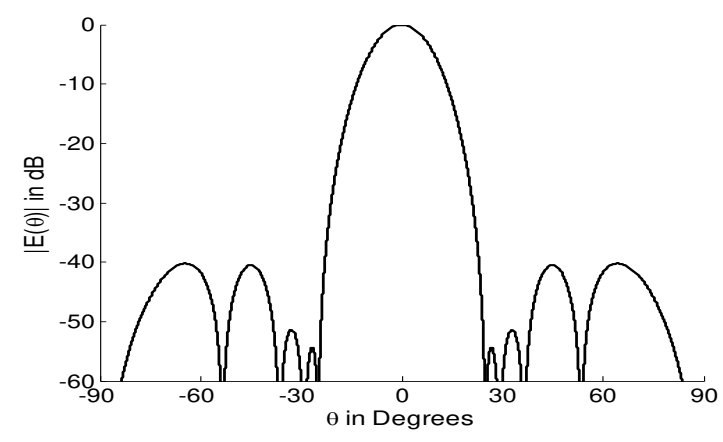

Fig. 5: 10 elements Radiation pattern with reduced close-in sidelobes to -50dB

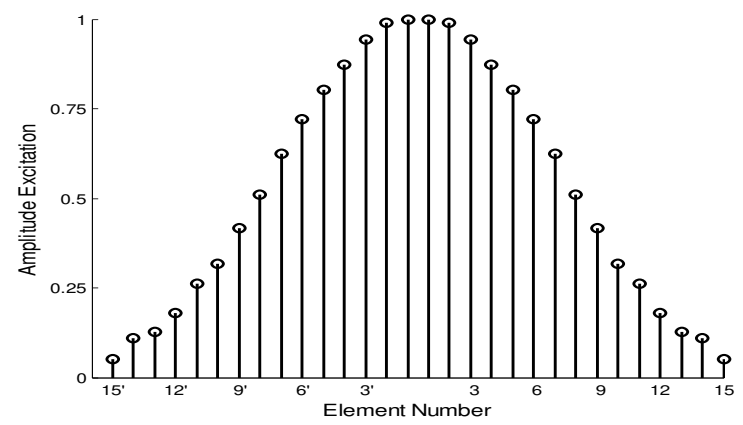

Fig 6: 30 elements amplitude distribution. 


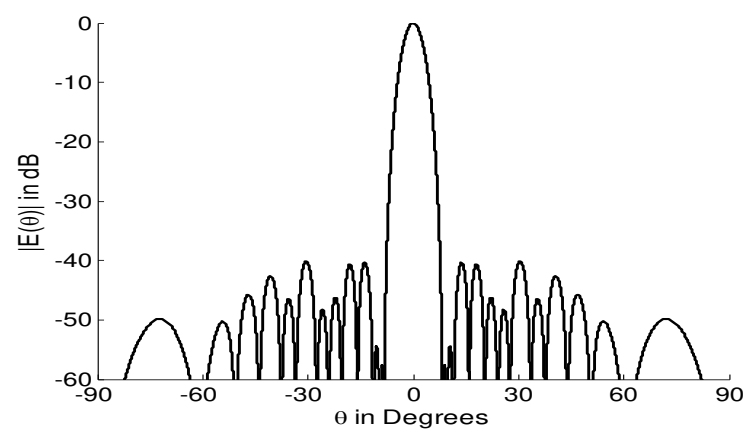

Fig. 7: 30 elements Radiation pattern with reduced close-in sidelobes to -50dB



Fig. 8: 50 elements amplitude distribution.

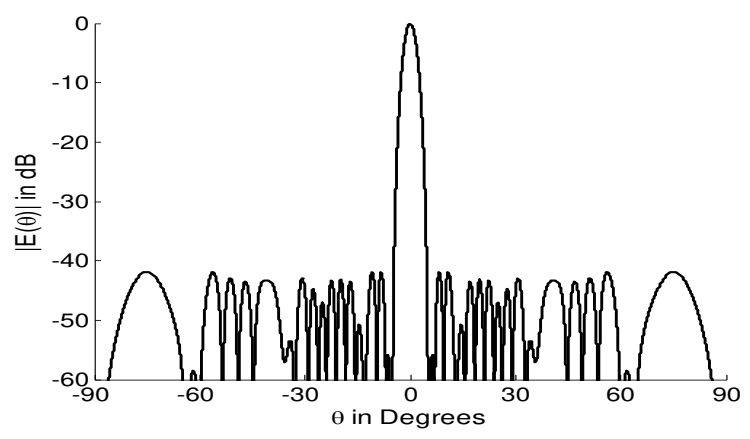

Fig. 9: 50 elements Radiation pattern with reduced close-in sidelobes to $-50 \mathrm{~dB}$

In case 2, amplitude and phase excitations obtained using EFPA to yield optimized radiation patterns with flat top beam allowing a maximum ripple of $1 \mathrm{~dB}$, and with maximum side lobe level less than $-25 \mathrm{~dB}$. For angular regions of $80^{\circ}, 100^{\circ}$ and $140^{\circ}$ the coefficients are determined using EFPA. Sector Beam patterns are numerically computed using the obtained excitation coefficient and are presented in Figures $[10-15]$. 

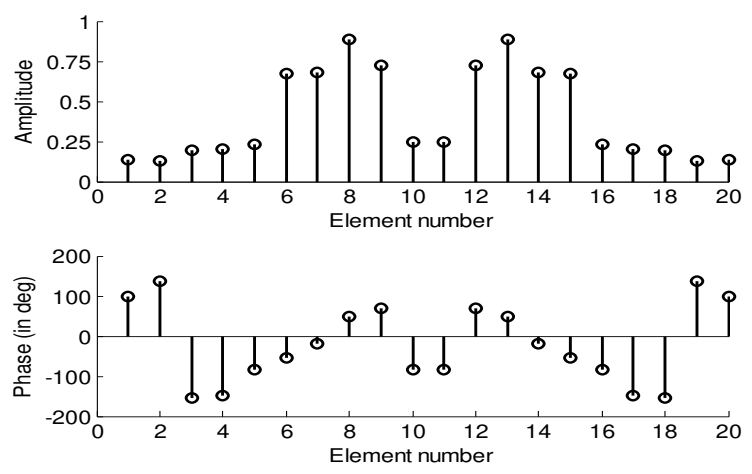

Fig. 10: For 20 Elements Amplitude and Phase Excitations



Fig. 11: Null to Null Beam width $=80^{\circ}$ for Sector Beam with 20 elements.
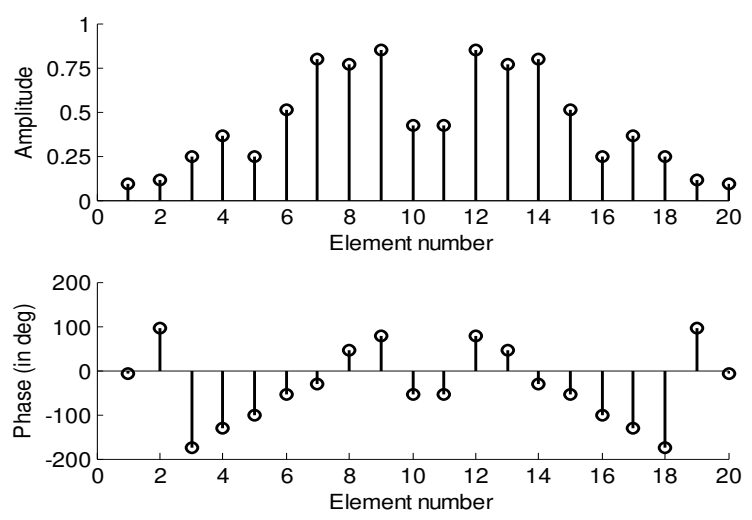

Fig. 12: For 20 Elements Amplitude and Phase Excitations 


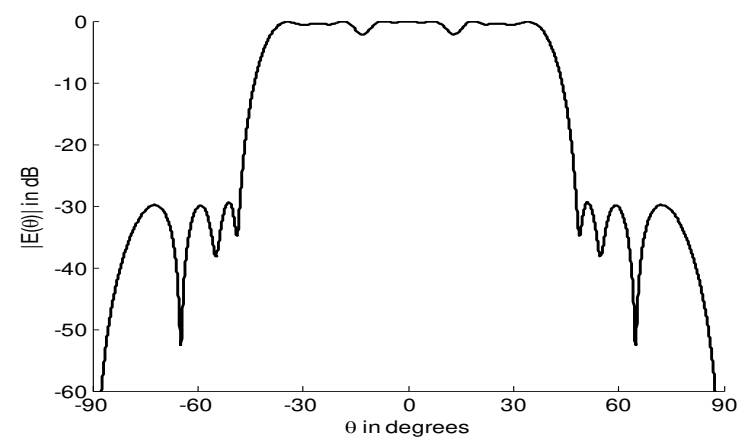

Fig. 13: Null to Null Beam width $=100^{0}$ for Sector Beam with 20 elements.
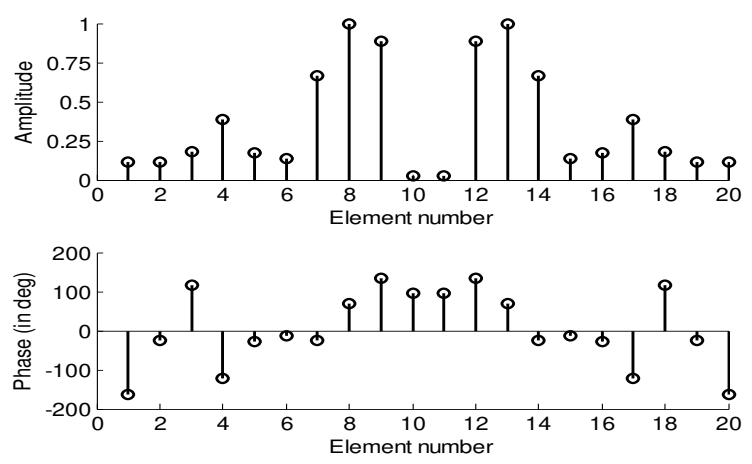

Fig. 14: For 20 Elements Amplitude and Phase Excitations

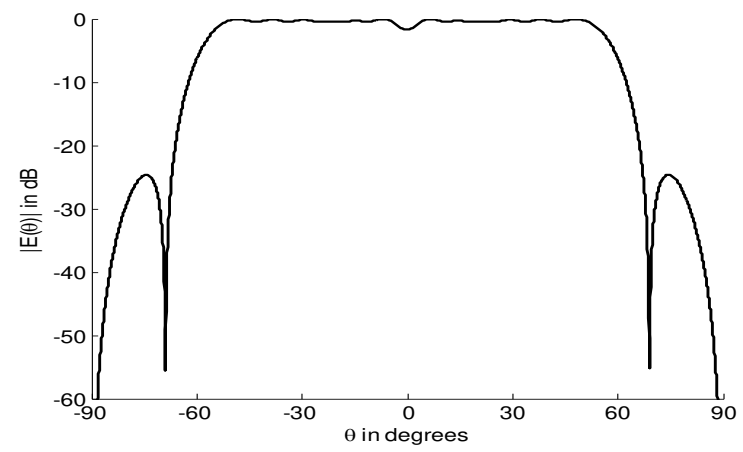

Fig. 15: Null to Null Beam width $=140^{\circ}$ for Sector Beam with 20 elements.

\section{CONCLUSION}

Using Enhanced Flower Pollination Algorithm, linear array synthesis is presented in the paper for a specified far-field sidelobe envelope. It is evident from the results that the lowest close-in side lobe level has been obtained without deteriorating the beam width. These patterns are very much useful for high resolution radars. The results of the analysis show that using optimum amplitude and phase distributions we can generate sector patterns. EFPA is applied to attain the required excitation coefficients. The results show considerably optimized sector beams using this technique. The ripples are well controlled and the sidelobe levels are maintained within acceptable limits. 
Compliance with Ethical Standards:

Funding: This research received no financial support.

Conflict of Interest: The authors declare that they have no conflict of interest.

Research involving human participants and/or animals:

This article does not contain any studies with human participants performed by any of the authors.

Authors Contribution:

Nagavalli Vegesna and Dr. Sudheer Kumar conceived the problem statement and the formulation presented in the paper. The Literature survey, simulation which involves in coding has been contributed by Dr. Sudheer Kumar. The simulation based experimentation, execution of the codes according to required format has been done by Dr G Yamuna. The Analysis and validation of the data has been performed by Dr. G Yamuna. The Manuscript preparation, data collection and other research facilities essential to carry out the work are provided by Nagavalli Vegesna and Dr. Sudheer Kumar.

\section{References}

1. Ram, G., Mandal, D., Kar, R., \&Ghoshal, S. P. (2015). Cat swarm optimization as applied to time-modulated concentric circular antenna array: Analysis and comparison with other stochastic optimization methods. IEEE transactions on Antennas and Propagation, 4180-4183.

2. Zhang, Z., Li, T., Yuan, F., \& Yin, L. (2014). Synthesis of linear antenna array using genetic algorithm to control side lobe level. In Computer engineering and networking (pp. 39-46). Springer, Cham.

3. Das, A., Mandal, D., Ghoshal, S. P., \&Kar, R. (2019). An optimal mutually coupled concentric circular antenna array synthesis using ant lion optimization. Annals of Telecommunications, 687696.

4. Amaireh, A. A., Al-Zoubi, A. S., \& Dib, N. I. (2019). Sidelobe-level suppression for circular antenna array via new hybrid optimization algorithm based on antlion and grasshopper optimization algorithms. Progress In Electromagnetics Research, 49-63.

5. Khodier, M. (2013). Optimisation of antenna arrays using the cuckoo search algorithm. IET Microwaves, Antennas \& Propagation, 458-464.

6. Liang, S., Feng, T., \& Sun, G. (2017). Sidelobe-level suppression for linear and circular antenna arrays via the cuckoo search-chicken swarm optimisation algorithm. IET Microwaves, Antennas \& Propagation, 209-218.

7. Saxena, P., \& Kothari, A. (2016). Linear antenna array optimization using flower pollination algorithm. SpringerPlus, 1-15. 
8. Rahman, S. U., CAO, Q., Ahmed, M. M., \& Khalil, H. (2017). Analysis of linear antenna array for minimum side lobe level, half power beamwidth, and nulls control using PSO. Journal of Microwaves, Optoelectronics and Electromagnetic Applications, 577-591.

9. Yigit, M. E., \&Gunel, T. (2018, October). Pattern synthesis of linear antenna array via a new hybrid Taguchi-genetic-particle swarm optimization algorithm. In 2018 18th Mediterranean Microwave Symposium (MMS) (pp. 17-21). IEEE.

10. Almagboul, M. A., Shu, F., Qian, Y., Zhou, X., Wang, J., \& Hu, J. (2019). Atom search optimization algorithm based hybrid antenna array receive beamforming to control sidelobe level and steering the null. AEU-International Journal of Electronics and Communications, 111, 152854.

11. Sun, G., Liu, Y., Li, H., Liang, S., Wang, A., \& Li, B. (2018). An antenna array sidelobe level reduction approach through invasive weed optimization. International Journal of Antennas and Propagation.

12. Grewal, N. S., Rattan, M., \&Patterh, M. S. (2012). A linear antenna array failure correction using firefly algorithm. Progress in Electromagnetics Research, 241-254.

13. Yoshimoto, E., \& Heckler, M. V. (2019). Optimization of planar antenna arrays using the firefly algorithm. Journal of Microwaves, Optoelectronics and Electromagnetic Applications, 126-140.

14. Singh, U., Salgotra, R., \& Rattan, M. (2016). A novel binary spider monkey optimization algorithm for thinning of concentric circular antenna arrays. IETE Journal of Research, 736-744.

15. Recioui, A., Traikia, O., \&Grainat, Y. (2019). Elliptic and Concentric-Elliptic Antenna Array Design using Grey Wolf Optimization. Algerian Journal of Signals and Systems, 32-38.

16. Van Luyen, T., \&Giang, T. V. B. (2017). Interference suppression of ULA antennas by phaseonly control using bat algorithm. IEEE Antennas And Wireless Propagation Letters, 3038-3042.

17. Mirjalili, S., \& Lewis, A. (2016). The whale optimization algorithm. Advances in engineering software, 51-67.

18. Zhang, C., Fu, X., Ligthart, L. P., Peng, S., \&Xie, M. (2018). Synthesis of broadside linear aperiodic arrays with sidelobe suppression and null steering using whale optimization algorithm. IEEE antennas and wireless propagation letters, 347-350.

19. Guney, K., Babayigit, B. İ. L. A. L., \&Akdagli, A. (2007). Position only pattern nulling of linear antenna array by using a clonal selection algorithm (CLONALG). Electrical Engineering, 147153.

20. Akdagli, A., Guney, K., \&Babayigit, B. İ. L. A. L. (2007). Clonal selection algorithm for design of reconfigurable antenna array with discrete phase shifters. Journal of Electromagnetic Waves and Applications, 215-227.

21. Merad, L.; Bendimerad, F.; Meriah, S.(2008): Design of linear antenna arrays for side lobe reduction using the tabu search method. Int. Arab J. Inf. Technol, 219-222. 